\title{
Sartans and Melanoma: Valsartan Induced Multiple Primary Cutaneous Melanomas: first Description in the Medical Literature!
}

\author{
Georgi Tchernev*, Gavrail Poterov, Valeri Malev \\ Onkoderma- Clinic for Dermatology, Venereology and Dermatologic Surgery, General Skobelev, Sofia, Bulgaria
}

\begin{abstract}
Received: October 06, 2020; Accepted: October 09, 2020; Published: October 12, 2020
*Corresponding author: Professor Georgi Tchernev, PhD, Onkoderma-Clinic for Dermatology, Venereology and Dermatologic Surgery, Sofia, Bulgaria, Tel. No: 00359885588424; E-mail: georgi_tchernev@yahoo.de
\end{abstract}

\begin{abstract}
The data in the medical literature about the possible development of cutaneous melanoma and dysplastic nevi after therapy with sartans in the world literature are already dozens. The role of the renin angiotensin system in various cancers such as melanoma but breast cancer also seems to be discussed in the past, in the present, but seems to be still the subject of many future discussions, which do not have a definitive solution.

We describe the first case of multiple cutaneous melanomas in the world literature, which developed simultaneously after the introduction of systemic antihypertensive therapy with Valsartan. Given the already established role of the renin angiotensin system in melanogenesis, as well as the possibility of promoting carcinogenesis through the practical influence of the "pure substance" of sartans, their widespread use in the treatment of hypertension should be seriously debated.
\end{abstract}

Keywords: Melanoma, Melanogenesis; Antihypertensive Therapy; Valsartan, Sartans; Surgery; Multiple Primary Melanomas

\section{Introduction}

The available in vitro data in the world literature do not raise any doubt as to its definiteness that sartans affect melanogenesis and, depending on the location and type of the corresponding angiotensin receptors in the tumor tissue, are able not only to block but also to potentiate carcinogenesis and in particular the development of melanoma metastasis $[1,2]$. The role of the renin angiotensin system in various other cancers such as breast cancer e.g. is discussed in the past, as this fact is still the subject of many discussions, which do not find (for reasons unknown at the moment and for all of us) still a definitive solution or change of approach before the introduction of systemic antihypertensive therapy with sartans [3,4]. In vivo data on metastatic melanomas, cutaneous melanomas and dysplastic nevi after therapy with sartans in the world literature are already dozens [5-10].

\section{Case report}

The anamnesis is taken according to the patient's data and the accompanying documentation. We present a 65-year-old patient with arterial hypertension and prescription of complaints from 2013. On systemic treatment with Valsartan $160 \mathrm{mg}$ (1-0-1) for the period from 2013-2017, and in 2017 the medication was stopped due to loss of effectiveness (according to the anamnestic data). For the period 2013-2017 the patient has taken two different drugs / from different companies, in equivalent doses - twice a day $160 \mathrm{mg}$ single dose or $320 \mathrm{mg}$ total dose per day. According to the anamnestic data, the patient notices the appearance of a new pigmented lesion / de-novo emerged lesion in the period between $2015 / 2017$ or between two to 4 years after the start of systemic treatment with Valsartan (figure 1a, 1b). In the next two years - 2017-2019, the lesion increases in size and that is the reason for visiting a surgeon.

On November 6, 2019, the patient a surgery was performed in the General and Abdominal Surgery Clinic at the National Oncology Hospital on the occasion of the newly emerged pigmented lesion in the left temporal area (figure 1c). The available histopathological results were indicative of cutaneous melanoma with a lesion diameter of $1 \mathrm{~cm}$, Breslow tumor thickness of $3 \mathrm{~mm}$, Clark 4, with evidence of ulceration, no evidence of perineural infiltration and lymphovascular invasion, stage pT3bN0M0. The surgical margins were without tumor infiltration. The patient was presented to an oncology committee with a decision for staging CT and assessment for subsequent treatment. From the MRT performed on 11.12.19 the conclusion is: no visible secondary lesions in the head and neck, no lymphadenomegaly, mild cerebral atrophy. Sentinel lymph node was not performed due to a faulty initial resection field, namely: removal of the lesion with an initial surgical safety field of $2.5 \mathrm{~cm}$ in all directions and not with the recommended surgical field of $0.5 \mathrm{~cm}$ recommended on AJCC guidelines. The patient was left for initial follow-up. On 17.01.2020 PET-CT was performed with a conclusion: there are no data on the spread of the underlying disease. There are no data on skin lesions with metastatic activity. The recommendation is again to monitor small nodular lesions in the lungs, which are currently of unclear dignity. 


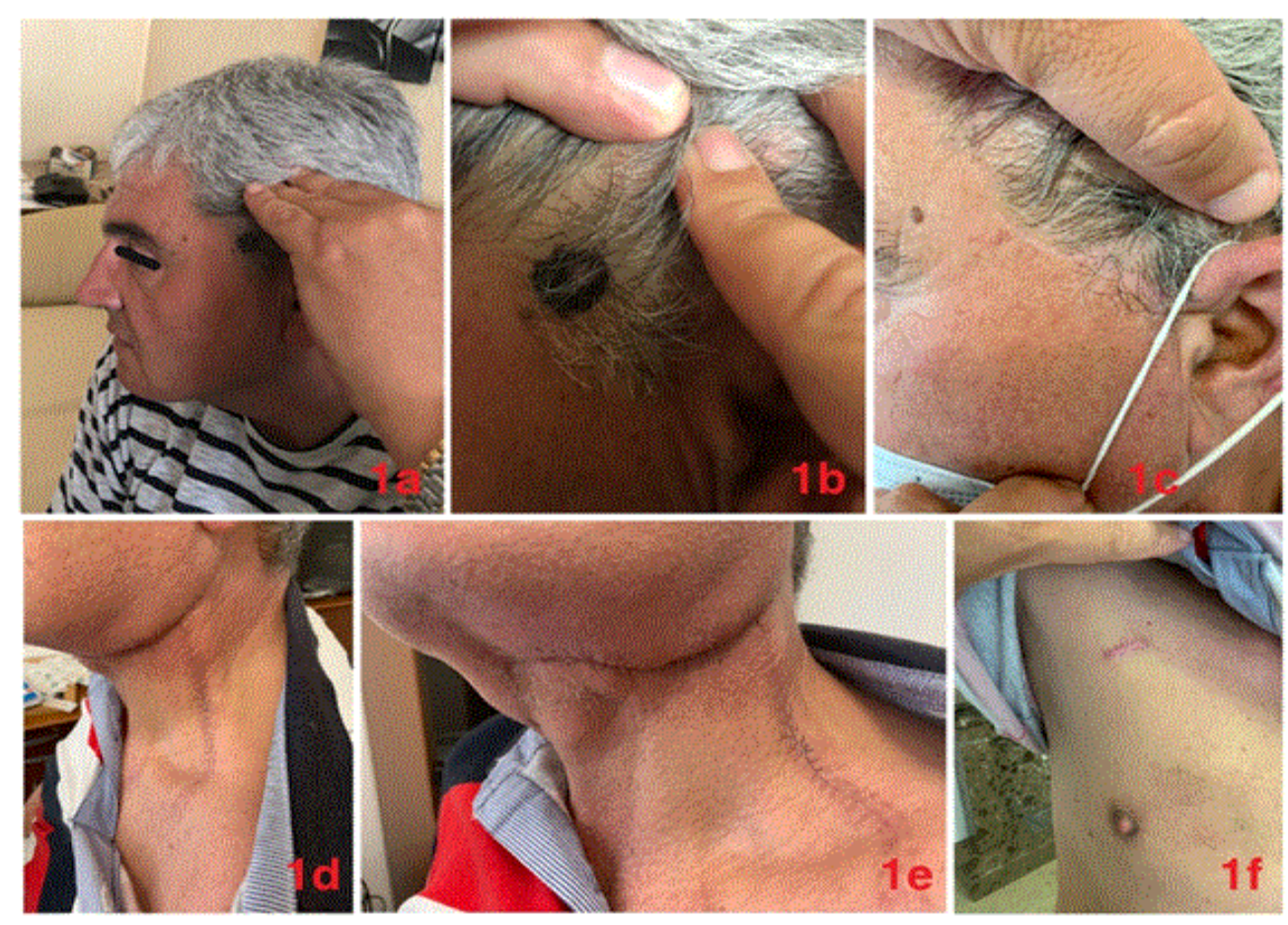

Figure 1: a) A newly appeared pigmented lesion in the area of the scalp, histopathologically subsequently proven as cutaneous melanoma. 1b)Cutaneous melanoma occurring several years after starting antihypertensive therapy with Valsartan. Preauricular localization

1c) Postoperative findings after surgical removal of the lesion

1d) - 1e) Clinical condition after modified cervical lymph dissection

1f) Postoperative cicatrix after removal of the second melanocytic lesion subsequently verificated histopathologically as thin cutaneous melanoma

In January 2020, as part of the clinical examinations, a second pigmented lesion was found on the right, contralateral to the previous one, and on 28.01.2020 the patient underwent reoperation and histopathological verification of the lesion showed evidence of thin skin melanoma: Breslow $0.1 \mathrm{~mm}$, Clark 1 (Figure 1f). On 16.04.2020 a PET-CT was performed again, within which data for the spread of the underlying disease were established. Presence of cervical lymph nodes on the left at levels IIa, IIb, III, VA, VB with moderate increased activity and type of metastatic.

The MRI performed on 05.05.2020 showed data on disease progression compared to the previous finding: suspicion of increased number and size of metastatic lymph nodes. There is suspected solitary lymph node from level IB on the right and additional data on lymph node involvement at level IV. The patient is recommended cervical lymph node dissection in view of suspected spread of the underlying disease.

He entered the clinic for maxillofacial surgery for cervical lymphadenopathy related to the main disease - metastatic malignant melanoma. A classical modified radical lymphatic dissection type III (Figure 1d, 1e) was performed (under general anesthesia) in the maxillofacial surgery department. 73 lymph nodes were found to be involved in the frame of the underlying disease and a total of 102 lymph nodes were surgically removed. Testing for BRAF from 08.06.2020 gives a positive result.
Transition to pT3bN3cM0 stage after lymph dissection. The patient was referred to the regional oncology dispensary to discuss the possibilities for targeted immunotherapy.

\section{Discussion}

It is unclear at this time and in the future whether initiating therapy with angiotensin receptor blockers should be accompanied by screening testing to assess the individual risk of possible development of melanoma and breast cancer, for example. Two socially and health-significant diseases with increasing frequency, for which the role of the renin angiotensin system in the tumor tissue is already real or experimentally proven [1-3]. An additional aggravating factor when starting treatment with sartans is the (in) ability to detect up to 3 nitrosamines as contaminants in the manufacturing process, which in its turn, according to FDA / EMA data, are able to lead to an additional 11 cancers forms [6]. Against the background of shared scientific and clinical data, the plot: "Don't worry, ask your cardiologist!" acquires rather or definitely grotesque meaning! We describe the first case of multiple cutaneous melanoma in the world literature, which developed simultaneously after the introduction of systemic antihypertensive therapy with Valsartan. Given the already established role of the renin angiotensin system in melanogenesis, as well as the possibility of promoting carcinogenesis through the practical influence of the "pure 
substance" of sartans, their widespread use in the treatment of hypertension should be seriously debated [1,2].

In the case described by us, important facts are presented, which should be subjected to a more in-depth future analysis:

1) Developing of de novo melanoma after starting of Valsartan therapy, already described by our team, but in another patient [6]

2) Developing of a second de novo melanoma after starting treatment with Valsartan, which is practically the first case in the world literature.

3) Development of melanoma metastases after Valsartan therapy, which is supported by the available serious experimental literature data [1,2], but also by the clinical ones [6].

4) Development of metastatic melanoma at a dose of valsartan of $320 \mathrm{mg}$, which is again described in the world literature, and the dose could be considered as a possible risk factor [6].

\section{References}

1. Renziehausen A, Wang H, Rao B, Weir L, Nigroet C, Lattanzio L, et al. The renin angiotensin system (RAS) mediates bifunctional growth regulation in melanoma and is a novel target for therapeutic intervention. Oncogene.2019;38(13):2320-2336. doi:10.1038/ s41388-018-0563-y

2. Olschewski DN, Hofschroer V, Nielsen N, Seidler DG, Schwab A, Stock C. The Angiotensin II Type 1 Receptor Antagonist Losartan Affects NHE1-Dependent Melanoma Cell Behavior. Cell Physiol Biochem. 2018;45(6):2560-2576. doi:10.1159/000488274

3. Acconcia F. The Network of Angiotensin Receptors in Breast Cancer. Cells. 2020;9(6):1336. doi:10.3390/cells9061336

4. Perini MV, Dmello RS, Nero TL, Chand AL. Evaluating the benefits of renin-angiotensin system inhibitors as cancer treatments. Pharmacol Ther. 2020;211:107527. doi:10.1016/j.pharmthera.2020.107527

5. Tchernev G, Temelkova I. Additional 4 cases of valsartan/irbesartaninduced melanomas? J Biol Regul Homeost Agents. 2019;33(3):911912.
6. Tchernev G, Temelkova I. Valsartan Induced Melanoma?! First Description in Medical Literature! Open Access Maced J Med Sci. 2018;6(12):2378-2380. doi:10.3889/oamjms.2018.517

7. Tchernev G, Temelkova I. Drug-Induced Melanoma: Irbesartan Induced Cutaneous Melanoma! First Description in the World Literature!. Open Access Maced J Med Sci. 2019;7(1):114-116. doi:10.3889/oamjms.2019.042

8. Tchernev G, Temelkova I. Irbesartan Induced Cutaneous Melanoma! Second Case in the Medical Literature! Open Access Maced J Med Sci. 2019;7(1):121-123. doi:10.3889/oamjms.2019.043

9. Tchernev G, Temelkova I. Olmesartan/valsartan induced giant achromatic cutaneous melanoma: "modified" one-step surgical approach with favourable outcome. J Biol Regul Homeost Agents. 2019;33(6):1775-1777.

10. Malev V, Tchernev G. Dysplastic nevus and BCC development after antihypertensive therapy with Valsartan and Hydrochlorothiazide!? Clin Res Dermatol Open Access. 2019;6(5):1-2. 